\title{
Development of the Colle-Salvetti correlation-energy formula into a functional of the electron density
}

\author{
Chengteh Lee, Weitao Yang, and Robert G. Parr \\ Department of Chemistry, University of North Carolina, Chapel Hill, North Carolina 27514
}

(Received 20 May 1987; revised manuscript received 27 August 1987)

\begin{abstract}
A correlation-energy formula due to Colle and Salvetti [Theor. Chim. Acta 37, 329 (1975)], in which the correlation energy density is expressed in terms of the electron density and a Laplacian of the second-order Hartree-Fock density matrix, is restated as a formula involving the density and local kinetic-energy density. On insertion of gradient expansions for the local kinetic-energy density, density-functional formulas for the correlation energy and correlation potential are then obtained. Through numerical calculations on a number of atoms, positive ions, and molecules, of both open- and closed-shell type, it is demonstrated that these formulas, like the original ColleSalvetti formulas, give correlation energies within a few percent.
\end{abstract}

\section{INTRODUCTION}

Interest in calculation of the correlation energy continues unabated. ${ }^{1,2}$ We here report a new development, in which the Colle-Salvetti formula for calculating correlation energy from the Hartree-Fock second-order density matrix ${ }^{3}$ is converted into a density-functional formula.

Let $\rho(\mathbf{r})$ be the Hartree-Fock electron density and $\rho_{2}^{\mathrm{HF}}\left(\mathbf{r}_{1}, \mathbf{r}_{2}\right)$ be the diagonal element of the second-order HartreeFock reduced density matrix,

$\rho_{2}^{\mathrm{HF}}\left(\mathbf{r}_{1}, \mathbf{r}_{2}\right)=\frac{N(N-1)}{2} \int \cdots \int\left|\Psi^{\mathrm{HF}}\left(\mathbf{r}_{1} \sigma_{1} \mathbf{r}_{2} \sigma_{2} \ldots\right)\right|^{2} d \sigma_{1} d \sigma_{2} \cdots d \sigma_{N} d \mathbf{r}_{3} d \mathbf{r}_{4} \cdots d \mathbf{r}_{N}$.

$\rho_{2}^{\mathrm{HF}}$ can also be expressed in terms of interparticle coordinates, $\rho_{2}^{\mathrm{HF}}(\mathbf{r}, \mathbf{s})$, where

$$
\mathbf{r}=\frac{\mathbf{r}_{1}+\mathbf{r}_{2}}{2}, \mathbf{s}=\mathbf{r}_{1}-\mathbf{r}_{2} .
$$

The Colle-Salvetti formula for correlation energy then is

$$
E_{c}=-4 a \int \frac{\rho_{2}^{\mathrm{HF}}(\mathbf{r}, \mathbf{r})}{\rho(\mathbf{r})}\left(\frac{1+b \rho(\mathbf{r})^{-8 / 3}\left[\nabla_{s}^{2} \rho_{2}^{\mathrm{HF}}(\mathbf{r}, \mathbf{s})\right]_{s=0} \exp \left[-c \rho^{-1 / 3}(\mathbf{r})\right]}{1+d \rho(\mathbf{r})^{-1 / 3}}\right) d \mathbf{r},
$$

where $a=0.04918, b=0.132, c=0.2533$, and $d=0.349$. This formula was arrived at by Colle and Salvetti by a theoretical analysis accompanied by a series of approximations, beginning (essentially) from the reasonable proposition that the second-order density matrix including correlation may approximated by the Hartree-Fock second-order density matrix times a correlation factor. The constants $a, b, c$, and $d$ in the final formula were obtained by a fitting procedure using only the HartreeFock orbital for the helium atom. Colle and Salvetti demonstrated that Eq. (3) gave good predicted correlation energies for atoms and molecules. ${ }^{3}$ They also provided and tested a generalized form for open-shell systems. ${ }^{4}$ Here we carry on the analysis of Eq. (3) itself.

\section{FORMULAS INVOLVING LOCAL KINETIC-ENERGY DENSITIES}

First consider a closed-shell atom or molecule, having an even number of electrons. Then
$\rho_{2}^{\mathrm{HF}}\left(\mathbf{r}_{1}, \mathbf{r}_{2}\right)=\frac{1}{2} \rho\left(\mathbf{r}_{1}\right) \rho\left(\mathbf{r}_{2}\right)-\frac{1}{4} \rho_{1}^{\mathrm{HF}}\left(\mathbf{r}_{1}, \mathbf{r}_{2}\right) \rho_{1}^{\mathrm{HF}}\left(\mathbf{r}_{2}, \mathbf{r}_{1}\right)$,

where $\rho_{1}^{\mathrm{HF}}$ is the Hartree-Fock first-order reduced density matrix,

$$
\begin{aligned}
\rho_{1}^{\mathrm{HF}}\left(\mathbf{r}_{1}, \mathbf{r}_{1}^{\prime}\right)=N \int \cdots \int & \Psi^{\mathrm{HF}^{*}}\left(\mathbf{r}_{1} \sigma_{1} \mathbf{r}_{2} \sigma_{2} \cdots\right) \\
& \times \Psi^{\mathrm{HF}}\left(\mathbf{r}_{1}^{\prime} \sigma_{1} \mathbf{r}_{2} \sigma_{2} \cdots\right) \\
& \times d \sigma_{1} d \sigma_{2} \cdots d \mathbf{r}_{2} d \mathbf{r}_{3} \cdots
\end{aligned}
$$

Note that $\rho_{1}^{\mathrm{HF}}(\mathbf{r}, \mathbf{r})=\rho(\mathbf{r})$ and $\rho_{2}^{\mathrm{HF}}(\mathbf{r}, \mathbf{r})=\frac{1}{4} \rho^{2}(\mathbf{r})$. In the interparticle coordinates of Eq. (2), (4) reads

$$
\begin{aligned}
\rho_{2}^{\mathrm{HF}}(\mathbf{r}, \mathbf{s})= & \frac{1}{2} \rho(\mathbf{r}+\mathbf{s} / 2) \rho(\mathbf{r}-\mathbf{s} / 2) \\
& -\frac{1}{4}\left[\rho_{1}^{\mathrm{HF}}(\mathbf{r}+\mathbf{s} / 2, \mathbf{r}-\mathbf{s} / 2)\right]^{2},
\end{aligned}
$$

and there follows 


$$
\left[\nabla_{s}^{2} \rho_{2}^{\mathrm{HF}}(\mathbf{r}, \mathbf{s})\right]_{s=0}=\rho(\mathbf{r})\left[t_{\mathbf{H F}}(\mathbf{r})-2 t_{W}(\mathbf{r})\right],
$$

where

$$
t_{W}(\mathbf{r})=\frac{1}{8} \frac{|\nabla \rho(\mathbf{r})|^{2}}{\rho(\mathbf{r})}-\frac{1}{8} \nabla^{2} \rho
$$

is a local "Weizsacker" kinetic-energy density and

$$
t_{\mathrm{HF}}(\mathbf{r})=\frac{1}{2}\left[\nabla_{r_{1}} \nabla_{r_{2}} \rho\left(\mathbf{r}_{1}, \mathbf{r}_{2}\right)\right]_{\mathrm{r}_{1}=\mathbf{r}_{2}=\mathbf{r}}-\frac{1}{8} \nabla^{2} \rho=\frac{1}{8} \sum_{i} \frac{\left|\nabla \rho_{i}(\mathbf{r})\right|^{2}}{\rho_{i}(\mathbf{r})}-\frac{1}{8} \nabla^{2} \rho
$$

is the local Hartree-Fock kinetic-energy density. Note that $t_{W}(\mathbf{r})$ and $t_{\mathrm{HF}}(\mathbf{r})$ as here defined include terms $-\frac{1}{8} \nabla^{2} \rho$ recommended for inclusion in other connections. ${ }^{5}$ To prove Eq. (7), operate on (6) with $\nabla_{s}^{2}=\frac{1}{4} \nabla_{r}^{2}-\nabla_{r_{1}} \cdot \nabla_{r_{2}}$ and take the $s=0$ limit.

Inserting Eq. (7) in Eq. (3), one obtains the correlation energy in terms of density, $t_{\mathrm{HF}}(\mathbf{r})$ and $t_{W}(\mathbf{r})$,

$$
E_{c}=-a \int \frac{\rho(\mathbf{r})+b \rho(r)^{-2 / 3}\left[t_{\mathrm{HF}}(\mathbf{r})-2 t_{W}(\mathbf{r})\right] e^{-c \rho(\mathbf{r})^{-1 / 3}}}{1+d \rho(\mathbf{r})^{-1 / 3}} d \mathbf{r}
$$

Results of calculations with this formula are given in Table I. For atoms, Clementi wave functions were used; ${ }^{6}$ for molecules, wave functions of Snyder and Basch. $^{7}$ These are in complete agreement with the results of Colle and Salvetti, as they should be since Eq. (10) follows directly from Eq. (3).

Consider next a system in a state described by a general single determinant, in which the $\alpha$-spin electron density differs from the $\beta$-spin electron density. (In the open-shell restricted Hartree-Fock case, which here is our primary concern, the $\alpha$-spin orbitals are the same as the $\beta$-spin orbitals in the closed-shell part, but an excess of $\alpha$ - or $\beta$-spin orbitals in the rest.) Equation (4) is replaced by

$$
\begin{aligned}
\rho_{2}^{\mathrm{HF}}\left(\mathbf{r}_{1}, \mathbf{r}_{2}\right)= & \frac{1}{2} \rho\left(\mathbf{r}_{1}\right) \rho\left(\mathbf{r}_{2}\right) \\
& -\frac{1}{2} \rho_{1, \alpha \alpha}^{\mathrm{HF}}\left(\mathbf{r}_{1}, \mathbf{r}_{2}\right) \rho_{1, \alpha \alpha}^{\mathrm{HF}}\left(\mathbf{r}_{2}, \mathbf{r}_{1}\right) \\
& -\frac{1}{2} \rho_{1, \beta \beta}^{\mathrm{HF}}\left(\mathbf{r}_{1}, \mathbf{r}_{2}\right) \rho_{1, \beta \beta}^{\mathrm{HF}}\left(\mathbf{r}_{2}, \mathbf{r}_{1}\right),
\end{aligned}
$$

so that

$$
\rho_{2}^{\mathrm{HF}}(\mathbf{r}, \mathbf{r})=\frac{1}{2} \rho^{2}(\mathbf{r})-\frac{1}{2}\left[\rho_{\alpha}^{2}(\mathbf{r})+\rho_{\beta}^{2}(\mathbf{r})\right],
$$

where $\rho_{\alpha}(\mathbf{r})$ and $\rho_{\beta}(\mathbf{r})$ are the $\alpha$-spin and $\beta$-spin electron densities. With $t_{\mathrm{HF}}^{\alpha}(\mathbf{r})$ and $t_{\mathrm{HF}}^{\beta}(\mathbf{r})$ the corresponding kinetic-energy densities in the sense of Eq. (9), and with

$$
\gamma(\mathbf{r})=2\left[1-\frac{\rho_{\alpha}^{2}(\mathbf{r})+\rho_{\beta}^{2}(\mathbf{r})}{\rho^{2}(\mathbf{r})}\right],
$$

Eq. (7) is replaced by

$$
\begin{gathered}
{\left[\nabla_{s}^{2} \rho_{2}^{\mathrm{HF}}(\mathbf{r}, \mathbf{s})\right]_{s=0}=2\left[\rho_{\alpha}(\mathbf{r}) t_{\mathrm{HF}}^{\alpha}(\mathbf{r})+\rho_{\beta}(\mathbf{r}) t_{\mathrm{HF}}^{\beta}(\mathbf{r})\right.} \\
\left.-\rho(\mathbf{r}) t_{W}(\mathbf{r})\right]
\end{gathered}
$$

and Eq. (10) by

$$
E_{c}=-a \int \frac{\rho(\mathbf{r})+2 b \rho(\mathbf{r})^{-5 / 3}\left[\rho_{\alpha}(\mathbf{r}) t_{\mathrm{HF}}^{\alpha}(\mathbf{r})+\rho_{\beta}(\mathbf{r}) t_{\mathrm{HF}}^{\beta}(\mathbf{r})-\rho(\mathbf{r}) t_{W}(\mathbf{r})\right] e^{-c \rho(\mathbf{r})^{-1 / 3}}}{1+d \rho(\mathbf{r})^{-1 / 3}} \gamma(\mathbf{r}) d \mathbf{r}
$$

Again numerical results obtained from the formula are very good; see Table I.

\section{GRADIENT EXPANSION FORMULAS}

Equations (10) and (15) lack of being pure density functionals only because of the appearance in them of the Hartree-Fock local kinetic-energy densities $t_{\mathrm{HF}}, t_{\mathrm{HF}}^{\alpha}$, and $t_{\mathrm{HF}}^{\beta}$, which depend on individual orbital densities. If the view is adopted that the orbitals are the Kohn-Sham orbitals, then these quantities too are functionals of the density, and Eqs. (10) and (15) are pure densityfunctional expressions [as would be Eq. (3) with this interpretation]. Eschewing that view for the present, one can turn Eqs. (10) and (15) into explicit density- functional formulas by using gradient expansions, whereby $t_{\mathrm{HF}}$ is expanded about the Thomas-Fermi local kinetic-energy density $t_{\mathrm{TF}}$.

The Thomas-Fermi kinetic-energy density is given by

$$
t_{\mathrm{TF}}(r)=C_{F} \rho(\mathbf{r})^{5 / 3}, \quad C_{F}=\frac{3}{10}\left(3 \pi^{2}\right)^{2 / 3} .
$$

In terms of this, the conventional closed-shell gradient expansion of $t_{\mathrm{HF}}$ gives, ${ }^{8}$ to zero order, ${ }^{9}$

$$
t_{\mathrm{HF}}(\mathbf{r})=t_{\mathrm{TF}}(\mathbf{r})=C_{F} \rho(\mathbf{r})^{5 / 3} ;
$$

to second order,

$$
t_{\mathrm{HF}}(\mathbf{r})=t_{\mathrm{TF}}(\mathbf{r})+\left[\frac{1}{9} t_{W}(\mathbf{r})+\frac{1}{18} \nabla^{2} \rho\right],
$$


and so on. An alternative formula is provided by the mean-path approximation, ${ }^{10}$ to zero order,

$$
t_{\mathrm{HF}}(\mathbf{r})=t_{\mathrm{TF}}(\mathbf{r})+\left[\frac{1}{9} t_{W}(\mathbf{r})-\frac{1}{36} \nabla^{2} \rho\right]
$$

For the open-shell case, the corresponding formulas are readily derived using ${ }^{11}$

$$
\begin{aligned}
& t_{\mathrm{HF}}^{\alpha}(\mathbf{r})=\frac{1}{2} t_{\mathrm{HF}}\left(2 \rho_{\alpha}(\mathbf{r}), \mathbf{r}\right), \\
& t_{\mathrm{HF}}^{\beta}(\mathbf{r})=\frac{1}{2} t_{\mathrm{HF}}\left(2 \rho_{\beta}(\mathbf{r}), \mathbf{r}\right) .
\end{aligned}
$$

The correlation energy formulas of Eqs. (10) and (15) then become

$$
E_{c}=-a \int \frac{1}{1+d \rho^{-1 / 3}}\left\{\rho+b \rho^{-2 / 3}\left[C_{F} \rho^{5 / 3}-2 t_{W}+\left(\frac{1}{9} t_{W}+\frac{1}{18} \nabla^{2} \rho\right)\right] e^{-c \rho^{-1 / 3}}\right\} d \mathbf{r}
$$

and

$$
\begin{aligned}
E_{c}=-a \int \frac{\gamma(\mathbf{r})}{1+d \rho^{-1 / 3}}\left\{\rho+2 b \rho^{-5 / 3}\right. & {\left[2^{2 / 3} C_{F} \rho_{\alpha}^{8 / 3}+2^{2 / 3} C_{F} \rho_{\beta}^{8 / 3}-\rho t_{W}\right.} \\
& \left.\left.+\frac{1}{9}\left(\rho_{\alpha} t_{W}^{\alpha}+\rho_{\beta} t_{W}^{\beta}\right)+\frac{1}{18}\left(\rho_{\alpha} \nabla^{2} \rho_{\alpha}+\rho_{\beta} \nabla^{2} \rho_{\beta}\right)\right] e^{-c \rho^{-1 / 3}}\right\} d \mathbf{r}
\end{aligned}
$$

As written, these are conventional second-order gradient expansion formulas, for closed and open shells, respectively. The zero-order formulas are obtained by striking out the terms within the parentheses in each case. The zero-order mean-path formula is obtained by replacing $\frac{1}{18}$ with $-\frac{1}{36}$ in these terms.

\begin{tabular}{|c|c|c|c|c|c|}
\hline Species & Expt. $^{a}$ & $\begin{array}{l}\text { Colle-Salvetti } \\
\text { formula }^{\mathrm{b}}\end{array}$ & $\begin{array}{l}\text { Conventional } \\
\text { zero-order } \\
\text { gradient } \\
\text { expansion }\end{array}$ & $\begin{array}{l}\text { Conventional } \\
\text { second-order } \\
\text { gradient } \\
\text { expansion }\end{array}$ & $\begin{array}{l}\text { Mean-path } \\
\text { zero order }\end{array}$ \\
\hline \multicolumn{6}{|c|}{ Closed shell } \\
\hline $\mathrm{He}$ & 0.0420 & $0.0416(0.0416)$ & 0.0390 & 0.0437 & 0.0394 \\
\hline $\mathrm{Li}^{+}$ & 0.0435 & $0.0438(0.0438)$ & 0.0388 & 0.0475 & 0.0385 \\
\hline $\mathrm{Be}^{2+}$ & 0.0443 & $0.0442(0.0441)$ & 0.0368 & 0.0490 & 0.0356 \\
\hline $\mathrm{Be}$ & $0.094^{\mathrm{f}}$ & $0.0926(0.0926)$ & 0.083 & 0.095 & 0.082 \\
\hline $\mathbf{B}^{+}$ & $0.111^{f}$ & $0.106(0.106)$ & 0.092 & 0.107 & 0.091 \\
\hline $\mathrm{Ne}$ & $0.387^{f}$ & $0.375(0.374)$ & 0.313 & 0.383 & 0.354 \\
\hline $\mathrm{Ar}$ & $0.79^{\mathrm{g}}$ & 0.743 & 0.710 & 0.751 & 0.702 \\
\hline $\mathrm{Kr}$ & & 1.735 & 1.683 & 1.748 & 1.668 \\
\hline $\mathrm{Xe}$ & & 2.733 & 2.659 & 2.743 & 2.640 \\
\hline $\mathrm{H}_{2} \mathrm{O}$ & $0.372^{\mathrm{h}}$ & $0.336(0.336)$ & 0.317 & 0.338 & 0.314 \\
\hline $\mathrm{CH}_{4}$ & $0.293^{t}$ & $0.290(0.289)$ & 0.275 & 0.294 & 0.273 \\
\hline \multicolumn{6}{|l|}{ Open shell } \\
\hline $\mathrm{Li}\left({ }^{2} S\right)$ & $0.045^{\mathrm{h}}$ & 0.050 & 0.045 & 0.053 & 0.045 \\
\hline$B\left({ }^{2} P\right)$ & $0.125^{\mathrm{h}}$ & 0.128 & 0.114 & 0.128 & 0.114 \\
\hline$C\left({ }^{3} P\right)$ & $0.156^{\mathrm{h}}$ & 0.161 & 0.146 & 0.161 & 0.146 \\
\hline $\mathbf{N}\left({ }^{4} S\right)$ & $0.189^{h}$ & 0.188 & 0.176 & 0.193 & 0.175 \\
\hline $\mathrm{Cl}\left({ }^{2} P\right)$ & $0.735^{\mathrm{h}}$ & 0.688 & 0.656 & 0.695 & 0.648 \\
\hline
\end{tabular}

Table I gives numerical results obtained from these formulas. The second-order gradient formulas are hardly distinguishable from the original Eqs. (10) and (15) and provide excellent representations of actual correlation energies. The conventional zero-order gradient formula and the zero-order mean-path formula are not quite as good.

TABLE I. Correlation energies of atoms and molecules. Negative correlation energies are given in atomic units. See text for calculational details.

${ }^{a}$ Exact energy corrected to be relatively free for infinite nuclear mass.

${ }^{b}$ Equation (10) or (15) of text. Valves in parentheses from Ref. 3.

${ }^{c}$ Equation (21) or (22) of text, without terms in parentheses.

${ }^{\mathrm{d}}$ Equation (21) or (22) of text.

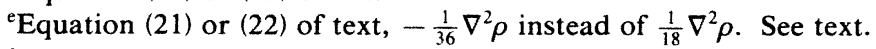

${ }^{\mathrm{f}}$ Reference 2 of text.

${ }^{g}$ E. Clementi, Tables of Atomic Functions, supplement to E. Clementi, IBM J. Res. Develop. 9, 2 (1965).

${ }^{\text {h}}$ D. Feller, C. M. Boyle, and E. R. Davidson, J. Chem. Phys. 86, 3424 (1987). 


\section{CORRELATION POTENTIAL}

The correlation potential is the functional derivative of $E c$ with respect to $\rho(r)$. Equation (21) yields, for the closed-shell case,

$$
\begin{aligned}
\frac{\hat{\delta} E_{c}}{\hat{\partial} \rho}= & -a\left(F_{1}^{\prime} \rho+F_{1}\right)-a b C_{F} \rho^{5 / 3}\left(G_{1}^{\prime} \rho+\frac{8}{3} G_{1}\right)-\frac{a b}{4}\left[G_{1}^{\prime \prime} \rho|\nabla \rho|^{2}+G_{1}^{\prime}\left(3|\nabla \rho|^{2}+2 \rho \nabla^{2} \rho\right)+4 G_{1} \nabla^{2} \rho\right] \\
& -\frac{a b}{72}\left[3 G_{1}^{\prime \prime} \rho|\nabla \rho|^{2}+G_{1}^{\prime}\left(5|\nabla \rho|^{2}+6 \rho \nabla^{2} \rho\right)+4 G_{1} \nabla^{2} \rho\right],
\end{aligned}
$$

where

$$
F_{1}(\rho)=\frac{1}{1+d \rho^{-1 / 3}}, \quad G_{1}(\rho)=F_{1}(\rho) \rho^{-5 / 3} e^{-c \rho^{-1 / 3}},
$$

$F_{1}^{\prime}$ (or $G_{1}^{\prime}$ ) is the first derivative of $F_{1}$ (or $G_{1}$ ) with respect to density, $\rho(r)$, and $G_{1}^{\prime \prime}$ is the second derivative of $G_{1}$ with respect to $\rho(r)$. For the open-shell case, the spin-dependent correlation potential obtained from Eq. (22) is

$$
\begin{aligned}
\frac{\hat{\partial} E_{c}}{\hat{\partial} \rho}= & -a\left(F_{2}^{\prime} \rho+F_{2}\right)-2^{5 / 3} a b C_{F}\left[G_{2}^{\prime}\left(\rho_{\alpha}^{8 / 3}+\rho_{\beta}^{8 / 3}\right)+\frac{8}{3} G_{2} \rho_{\alpha}^{5 / 3}\right]-\frac{a b}{4}\left[\rho \nabla^{2} G_{2}+4 \nabla G_{2} \cdot \nabla \rho+4 G_{2} \nabla^{2} \rho+G_{2}^{\prime}\left(\rho \nabla^{2} \rho-|\nabla \rho|^{2}\right)\right] \\
& -\frac{a b}{36}\left[3 \rho_{\alpha} \nabla^{2} G_{2}+4 \nabla \rho_{\alpha} \cdot \nabla G_{2}+4 G_{2} \nabla^{2} \rho_{\alpha}+3 G_{2}^{\prime}\left(\rho_{\alpha} \nabla^{2} \rho_{\alpha}+\rho_{\beta} \nabla^{2} \rho_{\beta}\right)+G_{2}^{\prime}\left(\left|\nabla \rho_{\alpha}\right|^{2}+\left|\nabla \rho_{\beta}\right|^{2}\right)\right],
\end{aligned}
$$

where

$$
F_{2}(\rho)=\frac{\gamma(\mathbf{r})}{1+d \rho^{-1 / 3}}, \quad G_{2}(\rho)=F_{2}(\rho) \rho^{-5 / 3} e^{-c \rho^{-1 / 3}}
$$

$F_{2}^{\prime}$ (or $G_{2}^{\prime}$ ) is the first derivative of $F_{2}$ (or $G_{2}$ ) with respect to $\rho_{\alpha}(r)$. The zero-order formulas for the correlation potential are obtained by omitting the last bracket- ed terms in these equations. Note that Eq. (25) reduces to Eq. (23) for the case $\rho_{\alpha}=\rho_{\beta}=\rho / 2$.

\section{DISCUSSION}

\begin{tabular}{|c|c|c|c|c|c|c|c|}
\hline Species & Colle-Salvetti $^{\mathrm{a}}$ & $\begin{array}{c}\text { Zero-order } \\
\text { gradient } \\
\text { expansion }^{\mathrm{b}}\end{array}$ & $\begin{array}{c}\text { Second-order } \\
\text { gradient } \\
\text { expansion }^{c}\end{array}$ & $V W N^{d, e}$ & $S P P^{d, f}$ & $G C L^{\mathrm{d}, \mathrm{g}}$ & $\mathrm{GCP}^{\mathrm{d}, \mathrm{h}}$ \\
\hline $\mathrm{He}$ & -0.0004 & -0.003 & 0.0017 & 0.07 & 0.017 & 0.004 & 0.003 \\
\hline $\mathrm{Li}^{+}$ & 0.0003 & -0.0047 & 0.004 & 0.092 & 0.026 & -0.013 & 0.0035 \\
\hline $\mathrm{Be}$ & -0.0014 & -0.011 & 0.001 & 0.131 & 0.022 & -0.006 & 0.001 \\
\hline $\mathbf{B}^{+}$ & -0.005 & -0.019 & -0.004 & 0.142 & 0.02 & -0.033 & -0.008 \\
\hline $\mathrm{Ne}$ & -0.012 & -0.074 & -0.004 & 0.359 & -0.001 & -0.009 & 0.008 \\
\hline $\mathrm{Li}$ & 0.005 & 0.00 & 0.008 & 0.106 & 0.027 & 0.006 & 0.009 \\
\hline B & 0.003 & -0.011 & 0.003 & 0.165 & 0.022 & -0.008 & 0.003 \\
\hline $\mathrm{C}$ & 0.005 & -0.01 & 0.005 & 0.203 & 0.02 & -0.006 & 0.009 \\
\hline $\mathbf{N}$ & -0.001 & -0.013 & 0.004 & 0.241 & 0.015 & -0.002 & 0.017 \\
\hline $\mathrm{H}_{2} \mathrm{O}$ & -0.036 & -0.055 & 0.034 & 0.292 & -0.028 & 0.011 & -0.007 \\
\hline $\mathrm{CH}_{4}$ & -0.003 & -0.018 & 0.001 & 0.3 & 0.014 & 0.079 & 0.035 \\
\hline
\end{tabular}

The Colle-Salvetti correlation energy formula, in the density-functional forms here derived, is seen to provide a good way to calculate correlation energies from elec-

TABLE II. Errors in correlation energies determined from various density-functional formulas, tabulated as values of $E_{\text {corr }}($ expt $)-E_{\text {corr }}$ (calc) for the various methods. For $E_{\text {corr }}$ (expt) values see Table I.

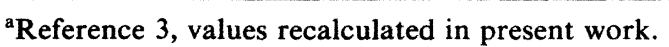

${ }^{\text {b}}$ Present work, Eq. (21) or (22) without term in parentheses.

${ }^{\mathrm{c}}$ Present work, Eq. (21) or (22) of text.

${ }^{\mathrm{d}}$ Numerical values from Ref. 2 of text.

${ }^{e}$ Method of S. H. Vosko, L. Wilk, and M. Nusair, Can J. Phys. 58, 1200 (1980).

${ }^{f}$ Method of H. Stoll, C. M. E. Pavlidou, and H. Preuss, Theor. Chim. Acta. 49, 143 (1978).

${ }^{8}$ Method of D. C. Langreth and D. J. Mehl, Phys. Rev. B 29, 2310 (1984).

${ }^{h}$ Method of Perdew, Ref. 1 of text. 
tron densities. Agreement with the calculated values of Perdew ${ }^{1}$ is close except for the species $\mathrm{Kr}$ and $\mathrm{Xe}$.

Table II gives detailed comparisons with other methods (extending a similar table in Ref. 2). The present second-order gradient expansion is seen to be not only an excellent approximation to the Colle-Salvetti formula, but at least as good as the other extant densityfunctional formulas. Also, there is a considerable computational advantage of the present formulas. The resultant Kohn-Sham exchange potentials contain no terms including the computationally awkward $\nabla|\nabla \rho|$.

The good numerical accuracy of the Colle-Salvetti formula, incorporating as it does only data on helium and theoretical arguments, commends it for further improvement and analysis. The predictive power can be improved by reparametrization using data on more systems. (Note for example that multiplying the parameter $a$ by $\frac{10}{9}$ greatly improves the zero-order results.) Also it may prove possible to correct for the deficiencies in the derivation.

\section{ACKNOWLEDGMENT}

This work has been aided by research grants from the National Institute of Health and the National Science Foundation to the University of North Carolina.
1J. P. Perdew, Phys. Rev. B 33, 8822 (1986).

${ }^{2}$ A. Savin, H. Stoll and H. Preuss, Theor. Chim. Acta 70, 407 (1986).

${ }^{3}$ R. Colle and D. Salvetti, Theor. Chim. Acta 37, 329 (1975). For the application to excited states, see R. Colle, R. Montagnani, P. Riani, and O. Salvetti, Theor. Chim. Acta. 49, 37 (1978). A later paper carries the analysis further: R. Colle and D. Salvetti, J. Chem. Phys. 79, 1404 (1983). For a comparative study, see L. Cohen and C. Lee, Int. J. Quantum Chem. Symp. 19, 535 (1986).

${ }^{4}$ R. Colle and D. Salvetti, Theor. Chim. Acta 53, 55 (1979). Also see R. Colle, F. Moscardo, P. Riani, and O. Salvetti, Theor. Chim. Acta 44, 1 (1977).
${ }^{5}$ M. Berkowitz, Chem. Phys. Lett. 129, 486 (1986); C. Lee and R. G. Parr, Phys. Rev. A 35, 2377 (1987).

${ }^{6}$ E. Clementi and C. Roetti, At. Data Nucl. Data Tables 14, 177 (1974).

${ }^{7}$ L. C. Snyder and H. Basch, Molecular Wave Functions and Properties (Wiley, New York, 1972).

${ }^{8}$ C. A. Hodges, Can. J. Phys. 51. 1428 (1973).

${ }^{9}$ Note from Eqs. (9) and (17) that a term $-\frac{1}{8} \nabla^{2} \rho$ is included in the zero-order gradient expansions. See Eq. (64) of Ref. 10, and W. Yang, R. G. Parr, and C. Lee, Phys. Rev. A 34, 4586 (1986).

${ }^{10}$ W. Yang, Phys. Rev. A 34, 4575 (1986).

${ }^{11}$ G. L. Oliver and J. P. Perdew, Phys. Rev. A 20, 397 (1979). 